\title{
A Strategy Plan for EPS
}

\begin{abstract}
The political and economic conditions are changing fast and a society like the EPS has to demonstrate its vitality by adapting to the changing outside world. Otherwise its raison d'être might be put in question. Hence it seems appropriate to review its activities and to decide on which issues to concentrate taking into account its limited resources. Council has approved a Strategy Plan which indicates those activities which should obtain high priority in the coming years. A dominating principle should be their complementarity to the activities of National Societies. Some of the activities in individual countries need an extension across Europe and some issues can only be dealt with on an international level.
\end{abstract}

EPS has been engaged in a number of varied activities carried out by Divisions, Interdivisional Groups, Action Committees and Task Forces. They have recently been reviewed by $\mathrm{H}$. Schopper [EN 26 (1995) 51] and J. Lewis [EN 26 (1995) 132]. Many of these "routine" long-term tasks have to continue, e.g., EPS and Divisional conferences, Europhysics News, qualification of conferences and journals (EPS "labels").

It is proposed to give high priority to not only these tasks but also to the following topics:

- Issues of professional physics

- Care for the next generation of physicists

- East-West cooperation

- Public awareness and information dissemination.

\section{Commentary to These Topics}

A few examples will be given to illustrate the four topics of priority.

\section{Issues of professional physics}

A number of issues relevant to the community of physicists deserves our attention. Among the most urgent are: - The relations between the academic world and physicists in industry need to be improved. The ties between research and technology have become closer and they will be decisive for the future competitiveness of Europe.

EPS should better communicate with physicists in industry, find out ways to give them support and maybe even seek closer contacts with industrial companies.

- The relations between physicists working at large facilities and in "small" science could be improved by a better exchange of information. Ways to achieve better mutual understanding and better cooperation and to develop a common policy for balanced support should be explored. This would increase the impact on funding authorities. - Promoting multidisciplinary activities (bio- and medical-physics, etc.).

- Training and teaching of physics (roads of access in academia and industry).

- Improving the ties with other scientific

European organizations, e.g., the European Science Foundation.

- The Register of "qualified physicists" and other measures to promote professional qualifications should be supported strongly.

Care for the next generation of physicists

For physics to survive (and with it EPS) it is of utmost importance to attract excellent young scientists and to give them appropriate support. This could be done by, for example:

- providing statistics of employment conditions and of the job market in Europe. The work of some national societies should be compiled and supplemented;

- supporting participation in international conferences and workshops;

- providing occasions to meet with industrialists;

- providing guidance on presentation when applying for jobs in an international environment;

- give them an active role in EPS activities.

\section{East-West cooperation}

East-West cooperation is entering a new era since the recent developments in various countries have evolved quite differently and require an individual approach. A new task force including members of east European countries to identify the most urgent needs has been established and must start its work. Special efforts concerning the exchange of physicists and their participation in international conferences and workshops is required.

\section{Public awareness and information dissemination}

Physics will be vital for society in the future, be it as part of human culture or as an indispensable source for technological progress. It is not only the results of physics research which are and will be essential (e.g., for energy production, distribution and saving, communication technology, transport, medicine), but also the methods developed for the exploration of Nature are extremely useful in other domains (e.g., mathematics, chemistry, biology, engineering, medicine and even sociology). Politicians and the public in general are not sufficiently aware of the importance of physics and even physicists when employed outside the physics domain. The following meas-

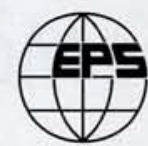

European Physical Society

Published by Europhysics News, the bulletin of EPS, to inform members. Editor: P.G. Boswell, EPS, BP 69, CH-1213, Petit-Lancy 2.

Tel +41-22-793 1130 - peter.boswell@cern.ch Fax +41-22-793 1317- http:/epswww.epfl.ch/

ures, among others, to improve the situa-

tion could be considered:

- EPS should make a considerable effort to improve public awareness by improving its relations with the media, and by organizing public conferences and exhibitions; - Studies of general importance involving physics should be carried out and the results published, e.g., on specific environmental problems, radioactivity, electrosmog;

- EPS should in particular become active in convincing governments, but above all the authorities of the European Union, to provide adequate support for physics in general and EPS activities in particular; - electronic communication and publishing will become increasingly important and EPS should take a lead,

- EPS should improve its own visibility.

All the questions and problems connected to these developments require international solutions. EPS could and should play an important role in finding satisfactory answers.

\section{Realization of the Strategic Objectives}

Council approved on 23 March the four topics of priority so the Executive Committee will work out a definitive Strategic Plan taking into account the discussion in Council and any additional suggestions made by National Societies or members of EPS.

To put into practice such a plan EPS will have to change its mode of operation. In view of the limited resources of the Secretariat, it will be necessary to contract to the outside some of the tasks. National Societies which have been engaged in some of the tasks or other European organizations with special competence might be prepared to carry out activities in cooperation with EPS ( maybe with some financial compensation). Other tasks might have to be allocated to outside firms or organizations. The necessary funds should be obtained from the savings arising from the move of the Secretariat, from improvement in the efficiency of some administrative operations and by securing of additional outside funds.

The Executive Committee 